\title{
Pairwise soft separation axioms in soft bigeneralized topological spaces
}

\author{
Adem Yolcu and Taha Yasin Ozturk \\ Department of Mathematics, Kafkas University, Kars, Turkey \\ Received: 7 August 2019, Accepted: 18 November 2019 \\ Published online: 26 December 2019.
}

\begin{abstract}
In this paper, we define and study some soft separation axioms in soft bigeneralized topological spaces in terms of pairwise soft bigeneralized $T_{i}$-spaces $(i=0,1,2,3,4)$. Characterizations and properties of these soft separation axioms have been obtained. Finally, we discuss the soft hereditary properties for these spaces.
\end{abstract}

Keywords: Soft set, Soft topology, Soft bigeneralized topology, Soft bigeneralized topological space, Soft separation axioms, Pairwise soft separation axioms.

\section{Introduction}

In the year 1999, D. Molodtsov [17] introduced the soft set theory as a new mathematical tools for dealing with uncertainties inherent in many of real world problems. Since a good number of papers is being published every year, soft set theory is getting popularity among the researchers $[13,14]$.

In 1963, N. Levine [12] tried to generalize a topology by replacing open sets with semi-open sets. Later, A. Csaszar [3] introduced the concept of $\gamma$-open sets and generalized these open sets. The theory of generalized topological spaces (briefly $G T$ ), which was founded by A. Csaszar [2] is one of the most important developments of general topology. Later, W. K. Min and Y. K. Kim [16] initiated the concept of bigeneralized topological spaces and quasi generalized open sets. In addition some separation axioms in bigeneralized topological spaces studied by P.Torton et al. [23].

Recently M. Shabir and M. Naz [20] initiated the study of soft topological spaces. Also they defined soft topology as a collection of soft sets over $X$ and they showed that soft topological spaces gives a parameterized family of topological spaces. After then many researchers studied on soft topological spaces [1,4,5,7,15,18,21,24]. J.C. Kelly [10], first initiated the concept of bitopological spaces. In 2014 B.M.Ittanagi [8] introduced the soft bitopological spaces which are defined over an initial universe with a fixed set of parameters. In later years, many researchers studied some of basic concept and properties on soft bitopological spaces [8,9].

In the year 2014, J.Thomas and S.J.John [22] initiated the concept of generalized topological spaces. They showed that a soft generalized topology gave a parameterized family of generalized topologies on the initial universe. Recently, In 2018, T. Y. Ozturk et. al. [19] introduced the soft bigeneralized topological spaces. Also they defined soft open set, soft closed set, soft closure set etc. on the soft bigeneralized topological spaces. Thus they showed that soft bigeneralized topological spaces are important generalized of bigeneralized topological spaces. In [6] paper, they studied soft 
generalized continuity, soft generalized open (closed) mapping and soft generalized homeomorphism on soft bigeneralized topological spaces.

In the present paper, we introduced some pairwise soft separation axioms in soft bigeneralized topological spaces. Characterizations and properties of these pairwise soft separation axioms have been obtained. Finally, we showed that soft hereditary properties of pairwise soft bigeneralized $T_{i}-$ spaces $(i=0,1,2,3,4)$.

\section{Preliminary}

In this section we will introduce necessary definitions and theorems for soft sets. Throughout this paper $X$ denotes initial universe, $E$ denotes the set of all parameters, $P(X)$ denotes the power set of $X$.

Definition 1. [17]. A pair $(F, E)$ is called a soft set over $X$, where $F$ is a mapping given by $F: E \rightarrow P(X)$.

In other words, the soft set is a parameterized family of subsets of the set $X$. For e $\in E, F(e)$ may be considered as the set of e-elements of the soft set $(F, E)$, or as the set of e-approximate elements of the soft set, i.e.,

$$
(F, E)=\{(e, F(e)): e \in E, F: E \rightarrow P(X)\} .
$$

After this, $S S(X)_{E}$ denotes the family of all soft sets over $X$ with a fixed set of parameters $E$.

Definition 2. [14]. For two soft sets $(F, E)$ and $(G, E)$ over $X,(F, E)$ is called a soft subset of $(G, E)$ if $\forall e \in E$, $F(e) \subseteq G(e)$. This relationship is denoted by $(F, E) \widetilde{\subseteq}(G, E)$.

Similarly, $(F, E)$ is called a soft superset of $(G, E)$ if $(G, E)$ is a soft subset of $(F, E)$. This relationship is denoted by $(F, E) \widetilde{\supseteq}(G, E)$. Two soft sets $(F, E)$ and $(G, E)$ over $X$ are called soft equal if $(F, E)$ is a soft subset of $(G, E)$ and $(G, E)$ is a soft subset of $(F, E)$.

Definition 3. [14]. The intersection of two soft sets $(F, E)$ and $(G, E)$ over $X$ is the soft set $(H, E)$, where $\forall e \in E$, $H(e)=F(e) \cap G(e)$. This is denoted by $(F, E) \widetilde{\cap}(G, E)=(H, E)$.

Definition 4. [14]. The union of two soft sets $(F, E)$ and $(G, E)$ over $X$ is the soft set $(H, E)$, where $\forall e \in E, H(e)=$ $F(e) \cup G(e)$. This is denoted by $(F, E) \widetilde{\cup}(G, E)=(H, E)$.

Definition 5. [14]. A soft set $(F, E)$ over $X$ is said to be a null soft set denoted by $(\widetilde{\phi}, E)$ if for all e $\in E, F(e)=\varnothing$.

Definition 6. [14]. A soft set $(F, E)$ over $X$ is said to be an absolute soft set denoted by $(\widetilde{X}, E)$ if for all $e \in E, F(e)=X$.

Definition 7. [20]. The difference $(H, E)$ of two soft sets $(F, E)$ and $(G, E)$ over $X$, denoted by $(F, E) \backslash(G, E)$, is defined as $H(e)=F(e) \backslash G(e)$ for all $e \in E$.

Definition 8. [20]. The complement of a soft set $(F, E)$, denoted by $(F, E)^{c}$, is defined $(F, E)^{c}=\left(F^{c}, E\right)$, where $F^{c}: E \rightarrow$ $P(X)$ is a mapping given by $F^{c}(e)=X \backslash F(e), \forall e \in E$ and $F^{c}$ is called the soft complement function of $F$.

Definition 9. [20]. Let $(F, E)$ be a soft set over $X$ and $Y$ be a non-empty subset of $X$. Then the sub soft set of $(F, E)$ over $Y$ denoted by $\left({ }^{Y} F, E\right)$, is defined as ${ }^{Y} F(\alpha)=Y \cap F(\alpha)$, for all $\alpha \in E$. In other words $\left({ }^{Y} F, E\right)=(Y, E) \cap(F, E)$.

Definition 10. [1]. Let $(F, E)$ be a soft set over $X$. The soft set $(F, E)$ is called a soft point, denoted by $\left(x_{e}, E\right)$, if for the element $e \in E, F(e)=\{x\}$ and $F\left(e^{c}\right)=\varnothing$ for all $e^{c} \in E-\{e\}$ (briefly denoted by $x_{e}$ ).

It is obvious that each soft set can be expressed as a union of soft points. For this reason, to give the family of all soft sets on $X$ it is sufficient to give only soft points on $X$. 
Definition 11. [1]. Two soft points $x_{e}$ and $y_{e^{\prime}}$ over a common universe $X$, we say that the soft points are different if $x \neq y$ or $e \neq e^{\prime}$.

Definition 12. [1]. The soft point $x_{e}$ is said to be belonging to the soft set $(F, E)$, denoted by $x_{e} \widetilde{\epsilon}(F, E)$, if $x_{e}(e) \in F(e)$, i.e., $\{x\} \subseteq F(e)$.

Definition 13. [1]. Let $(X, \widetilde{\tau}, E)$ be a soft topological space over $X$. A soft set $(F, E) \widetilde{\subseteq}(X, E)$ is called a soft neighborhood of the soft point $x_{e} \widetilde{\in}(F, E)$ if there exists a soft open set $(G, E)$ such that $x_{e} \widetilde{\in}(G, E) \widetilde{C}(F, E)$.

Definition 14. [22]. Let $\widetilde{\mu}$ be the collection of soft set over $X$. Then $\widetilde{\mu}$ is said to be a soft generalized topology on $X$ if

(1) $(\widetilde{\phi}, E)$ belongs to $\widetilde{\mu}$;

(2) the union of any number of soft sets in $\widetilde{\mu}$ belongs to $\widetilde{\mu}$.

The triplet $(X, \widetilde{\mu}, E)$ is called a soft generalized topological space (briefly SGT-space) over X.

Definition 15. [22]. A soft generalized topology $\widetilde{\mu}$ on $(X, E)$ is called strong if $\widetilde{X} \in \widetilde{\mu}$.

Definition 16. [22]. Let $(X, \widetilde{\mu}, E)$ be a SGT-space over $(X, E)$. Then every element of $\widetilde{\mu}$ is called a soft $g_{\widetilde{\mu}}-$ open set.

Definition 17. [22]. $(X, \widetilde{\mu}, E)$ be a SGT-space over $(X, E)$ and $(F, E) \widetilde{\subseteq}(X, E)$. Then $(F, E)$ is called a soft $g_{\widetilde{\mu}}$-closed set if its soft complement $(F, E)^{c}$ is a soft $g_{\tilde{\mu}}-$ open set.

Definition 18. [19]. Let $\widetilde{g}_{1}$ and $\widetilde{g}_{2}$ be two soft generalized topologies on $X$ and $E$ be a set of parameters. Then the quadruple system $\left(X, \widetilde{g}_{1}, \widetilde{g}_{2}, E\right)$ is called a soft bigeneralized topological space (briefly SBGT-space).

Definition 19. [19]. Let $\left(X, \widetilde{g}_{1}, \widetilde{g}_{2}, E\right)$ be a SBGT-space. A soft set $(F, E) \widetilde{\subseteq} X$ is called soft $\widetilde{g}_{1,2}-$ open set if $(F, E)=\left(F_{1}, E\right) \widetilde{\cup}\left(F_{2}, E\right)$ where $\left(F_{1}, E\right) \in \widetilde{g}_{1}$ and $\left(F_{2}, E\right) \in \widetilde{g}_{2}$.

The complement of soft $\widetilde{g}_{1,2}-$ open set is called soft $\widetilde{g}_{1,2}-$ closed set. Clearly, a soft set $(G, E)$ over $X$ is a soft $\widetilde{g}_{1,2}-$ closed set in $\left(X, \widetilde{g}_{1}, \widetilde{g}_{2}, E\right)$ if $(G, E)=\left(G_{1}, E\right) \widetilde{\cap}\left(G_{2}, E\right)$ such that $\left(G_{1}, E\right) \in \widetilde{g}_{1}^{c}$ and $\left(G_{2}, E\right) \in \widetilde{g}_{2}^{c}$, where

$$
\widetilde{g}_{i}^{c}=\left\{(G, E)^{c} \in S S(X)_{E}:(G, E) \in \widetilde{g}_{i}\right\}, i=1,2 .
$$

Definition 20. [19]. Let $\left(X, \widetilde{g}_{1}, \widetilde{g}_{2}, E\right)$ be a $S B G T$-space and $(F, E) \in S S(X)_{E}$. Then, soft $\widetilde{g}_{1,2}-$ closure set of $(F, E)$, denoted by $\operatorname{scl}_{\widetilde{g}_{1,2}}(F, E)$, defined by

$$
\operatorname{scl}_{\widetilde{g}_{1,2}}(F, E)=\widetilde{\cap}\left\{(G, E) \in \widetilde{g}_{1,2}^{c}:(F, E) \widetilde{\subseteq}(G, E)\right\} .
$$

Note that, $\operatorname{scl}_{\widetilde{g}_{1,2}}(F, E)$ is the smallest soft $\widetilde{g}_{1,2}$ - closed set that containing $(F, E)$.

Theorem 1. [19]. Let $\left(X, \widetilde{g}_{1}, \widetilde{g}_{2}, E\right)$ be a SBGT-space and $(F, E),(G, E) \in S S(X)_{E}$. Then,

(1) $\operatorname{scl}_{\widetilde{g}_{1,2}}(\widetilde{X}, E)=(\widetilde{X}, E)$,

(2) $(F, E) \widetilde{\subseteq} s c l_{\widetilde{g}_{1,2}}(F, E)$,

(3) $(F, E)$ is a soft $\widetilde{g}_{1,2}-$ closed set if and only if $\operatorname{scl}_{\widetilde{g}_{1,2}}(F, E)=(F, E)$,

(4) if $(F, E) \widetilde{\subseteq}(G, E)$, then $\operatorname{scl}_{\widetilde{g}_{1,2}}(F, E) \widetilde{\widetilde{\subseteq}} s_{\widetilde{g}_{1,2}}(G, E)$,

(5) $\operatorname{scl}_{\widetilde{g}_{1,2}}(F, E) \widetilde{\cup} s c{\widetilde{g}_{1,2}}_{(G, E) \widetilde{\subseteq}} s_{\widetilde{g}_{1,2}}[(F, E) \widetilde{\cup}(G, E)]$ and $\operatorname{scl}_{\widetilde{g}_{1,2}}[(F, E) \widetilde{\cap}(G, E)] \widetilde{\subseteq} s c l_{\widetilde{g}_{1,2}}(F, E) \widetilde{\cap} s c l_{\widetilde{g}_{1,2}}(G, E)$,

(6) $\operatorname{scl}_{\widetilde{g}_{1,2}}\left[\operatorname{scl}_{\widetilde{g}_{1,2}}(F, E)\right]=\operatorname{scl}_{\widetilde{g}_{1,2}}(F, E)$, i.e., $\operatorname{scl}_{\widetilde{g}_{1,2}}(F, E)$ is a soft $\widetilde{g}_{1,2}-$ closed set. 
Definition 21. [19]. Let $\left(X, \widetilde{g}_{1}, \widetilde{g}_{2}, E\right)$ be a SBGT-space and $(F, E) \in S S(X)_{E}$. Then, soft $\widetilde{g}_{1,2}-$ interior set of $(F, E)$, denoted by $\operatorname{sint}{\widetilde{\tilde{g}_{1,2}}}_{(}(F, E)$, defined by

$$
\operatorname{sint} \widetilde{g}_{1,2}(F, E)=\widetilde{\cup}\left\{(U, E) \in \widetilde{g}_{1,2}:(U, E) \widetilde{\widetilde{C}}(F, E)\right\} .
$$

Note that, sint $\widetilde{g}_{1,2}(F, E)$ is the largest soft $\widetilde{g}_{1,2}-$ open set contained in $(F, E)$.

Definition 22. [6]. Let $\left(X, \widetilde{g}_{1}, \widetilde{g}_{2}, E\right)$ and $\left(Y, \widetilde{k}_{1}, \widetilde{k}_{2}, E\right)$ be two SBGT-spaces and $\left(f, 1_{E}\right):\left(X, \widetilde{g}_{1}, \widetilde{g}_{2}, E\right) \rightarrow\left(Y, \widetilde{k}_{1}, \widetilde{k}_{2}, E\right)$ (briefly denoted by $f$ ) be a soft mapping. For each soft $\widetilde{k}_{1,2}-n b d(G, E)$ of $f\left(x_{e}\right)$, if there exists a soft $\widetilde{g}_{1,2}-n b d(F, E)$ of soft point $x_{e} \widetilde{\in} S S(X)_{E}$ such that $f((F, E)) \widetilde{\subseteq}(G, E)$, then $f$ is said to be soft $\widetilde{g}_{1,2}-$ continuous mapping at $x_{e}$.

If $f$ is a soft $\widetilde{g}_{1,2}$ - continuous mapping for all $x_{e} \widetilde{\in} S S(X)_{E}$, then $f$ is called a soft $\widetilde{g}_{1,2}-$ continuous mapping on a soft bigeneralized topological spaces $\left(X, \widetilde{g}_{1}, \widetilde{g}_{2}, E\right)$.

Definition 23. [6]. Let $\left(X, \widetilde{g}_{1}, \widetilde{g}_{2}, E\right)$ and $\left(Y, \widetilde{k}_{1}, \widetilde{k}_{2}, E\right)$ be two SBGT-spaces, $f:\left(X, \widetilde{g}_{1}, \widetilde{g}_{2}, E\right) \rightarrow\left(Y, \widetilde{k}_{1}, \widetilde{k}_{2}, E\right)$ be a soft mapping. Then,

(a) $f$ is called a soft $\widetilde{g}_{1,2}$ - open mapping if $f((F, E))$ is a soft $\widetilde{k}_{1,2}$ - open set in $\left(Y, \widetilde{k}_{1}, \widetilde{k}_{2}, E\right)$ for every soft $\widetilde{g}_{1,2}-$ open set $(F, E)$ in $\left(X, \widetilde{g}_{1}, \widetilde{g}_{2}, E\right)$;

(b) $f$ is called a soft $\widetilde{g}_{1,2}$ - closed mapping if $f((K, E))$ is a soft $\widetilde{k}_{1,2}-$ closed set in $\left(Y, \widetilde{k}_{1}, \widetilde{k}_{2}, E\right)$ for every soft $\widetilde{g}_{1,2}-$ closed set $(K, E)$ in $\left(X, \widetilde{g}_{1}, \widetilde{g}_{2}, E\right)$.

Theorem 2. [6]. Let $\left(X, \widetilde{g}_{1}, \widetilde{g}_{2}, E\right)$ and $\left(Y, \widetilde{k}_{1}, \widetilde{k}_{2}, E\right)$ be two $S B G T$-spaces and $f:\left(X, \widetilde{g}_{1}, \widetilde{g}_{2}, E\right) \rightarrow\left(Y, \widetilde{k}_{1}, \widetilde{k}_{2}, E\right)$ be a soft mapping. Then $f$ is a soft $\widetilde{g}_{1,2}$ - continuous mapping on a soft bigeneralized topological spaces $\left(X, \widetilde{g}_{1}, \widetilde{g}_{2}, E\right)$ iff $f^{-1}((G, E))$ is a soft $\widetilde{g}_{1,2}$ - open set, for every soft $\widetilde{k}_{1,2}$ - open set $(G, E)$.

Definition 24. [6]. Let $\left(X, \widetilde{g}_{1}, \widetilde{g}_{2}, E\right)$ and $\left(Y, \widetilde{k}_{1}, \widetilde{k}_{2}, E\right)$ be two SBGT-spaces, $f:\left(X, \widetilde{g}_{1}, \widetilde{g}_{2}, E\right) \rightarrow\left(Y, \widetilde{k}_{1}, \widetilde{k}_{2}, E\right)$ be a soft mapping. Then $f$ is called a soft $\widetilde{g}_{1,2}-$ homeomorphism, if

(i) $f$ is a soft bijection,

(ii) $f$ is a soft $\widetilde{g}_{1,2}$ - continuous,

(iii) $f^{-1}$ is a soft $\widetilde{g}_{1,2}$ - continuous mapping.

\section{Pairwise soft separation axioms}

Definition 25. Let $\left(X, \widetilde{g}_{1}, \widetilde{g}_{2}, E\right)$ be a SBGT-space over $X$ and each distinct soft points $x_{\alpha}, y_{\beta} \widetilde{\in} S S(X)_{E}$. If there exist a pairwise soft open $(F, E) \in \widetilde{g}_{12}$ such that $x_{\alpha} \widetilde{\in}(F, E), y_{\beta} \widetilde{\notin}(F, E)$ or $x_{\alpha} \tilde{\notin}(F, E), y_{\beta} \widetilde{\in}(F, E)$, then $\left(X, \widetilde{g}_{1}, \widetilde{g}_{2}, E\right)$ is called a pairwise soft bigeneralized $T_{0}$-space.

Example 1. Let $X=\{a, b\}, E=\left\{e_{1}, e_{2}\right\}$ and let

$$
\begin{aligned}
& \widetilde{g}_{1}=\left\{(\widetilde{\phi}, E),\left(F_{1}, E\right),\left(F_{2}, E\right)\right\} \\
& \widetilde{g}_{2}=\left\{(\widetilde{\phi}, E),\left(G_{1}, E\right),\left(G_{2}, E\right)\right\}
\end{aligned}
$$

where

$$
\begin{aligned}
\left(F_{1}, E\right) & =\left\{\left(e_{1},\{a\}\right),\left(e_{2}, \phi\right)\right\} \\
\left(F_{2}, E\right) & =\left\{\left(e_{1}, X\right),\left(e_{2},\{a\}\right)\right\} \\
\left(G_{1}, E\right) & =\left\{\left(e_{1},\{b\}\right),\left(e_{2}, \phi\right)\right\} \\
\left(G_{2}, E\right) & =\left\{\left(e_{1}, X\right),\left(e_{2},\{b\}\right)\right\}
\end{aligned}
$$


Then, $\left(X, \widetilde{g}_{1}, \widetilde{g}_{2}, E\right)$ is a SBGT-space. Thus, the family of pairwise soft open sets over $\left(X, \widetilde{g}_{1}, \widetilde{g}_{2}, E\right)$ becomes

$$
\widetilde{g}_{12}=\left\{(\widetilde{\phi}, E),\left(F_{1}, E\right),\left(F_{2}, E\right),\left(G_{1}, E\right),\left(G_{2}, E\right),\left(H_{1}, E\right),\left(H_{2}, E\right)\right\}
$$

where

$$
\begin{aligned}
& \left(H_{1}, E\right)=\left\{\left(e_{1}, X\right),\left(e_{2}, \phi\right)\right\} \\
& \left(H_{2}, E\right)=\left\{\left(e_{1}, X\right),\left(e_{2}, X\right)\right\}
\end{aligned}
$$

It is clear that $\left(X, \widetilde{g}_{1}, \widetilde{g}_{2}, E\right)$ is a pairwise soft bigeneralized $T_{0}-$ space.

Theorem 3. A SBGT-space $\left(X, \widetilde{g}_{1}, \widetilde{g}_{2}, E\right)$ is pairwise soft bigeneralized $T_{0}$-space if and only if $\operatorname{scl}_{\widetilde{g}_{12}}\left(x_{\alpha}, E\right) \neq \operatorname{scl}_{\widetilde{g}_{12}}\left(y_{\beta}, E\right)$ where each distinct soft points $x_{\alpha}, y_{\beta} \tilde{\in} S S(X)_{E}$.

Proof. Suppose that $\left(X, \widetilde{g}_{1}, \widetilde{g}_{2}, E\right)$ is pairwise soft bigeneralized $T_{0}$-space and each distinct soft points $x_{\alpha}, y_{\beta} \widetilde{\in} S S(X)_{E}$. Then, there exist $(G, E) \in \widetilde{g}_{12}$ such that $x_{\alpha} \widetilde{\in}(G, E), y_{\beta} \widetilde{\notin}(G, E)$. Hence $(G, E)^{c}$ is a soft $\widetilde{g}_{12}-$ closed set which contain $y_{\beta}$ but not $x_{\alpha}$. By Definition-20, $\operatorname{scl}_{\widetilde{g}_{12}}\left(y_{\beta}, E\right)$ is the smallest soft $\widetilde{g}_{12}-$ closed set that containing $\left(y_{\beta}, E\right)$. Therefore $\operatorname{scl}_{\widetilde{g}_{12}}\left(y_{\beta}, E\right) \widetilde{\subseteq}(G, E)^{c}$. So $x_{\alpha} \widetilde{\notin}(G, E)^{c}$ implies that $x_{\alpha} \widetilde{\notin} s c l_{\widetilde{g}_{12}}\left(y_{\beta}, E\right)$. Thus $x_{\alpha} \widetilde{\in} s c l_{\widetilde{g}_{12}}\left(x_{\alpha}, E\right)$ but $x_{\alpha} \widetilde{\notin} s c l_{\widetilde{g}_{12}}\left(y_{\beta}, E\right)$. Therefore $s c l_{\widetilde{g}_{12}}\left(x_{\alpha}, E\right) \neq \operatorname{scl}_{\widetilde{g}_{12}}\left(y_{\beta}, E\right)$.

Conversely, assume that each distinct soft points $x_{\alpha}, y_{\beta} \tilde{\in} S S(X)_{E}$. and $\operatorname{scl}_{\widetilde{g}_{12}}\left(x_{\alpha}, E\right) \neq \operatorname{scl}_{\widetilde{g}_{12}}\left(y_{\beta}, E\right)$. Then by assumption, there exist at least one soft point $z_{e} \widetilde{\in} S S(X)_{E}$ such that $z_{e} \widetilde{\in} s c l_{\widetilde{g}_{12}}\left(x_{\alpha}, E\right)$ but $z_{e} \widetilde{\notin}_{s c \widetilde{g}_{12}}\left(y_{\beta}, E\right)$. Now we show that

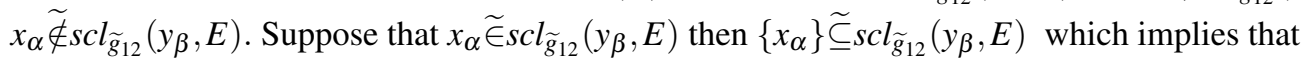
$\operatorname{scl}_{\widetilde{g}_{12}}\left(x_{\alpha}, E\right) \widetilde{\subseteq} s c l_{\widetilde{g}_{12}}\left(y_{\beta}, E\right)$. Hence $z_{e} \widetilde{\in} s c l_{\widetilde{g}_{12}}\left(x_{\alpha}, E\right)$ implies $z_{e} \widetilde{\in} s c l_{\widetilde{g}_{12}}\left(y_{\beta}, E\right)$. The contradicts the fact that $z_{e} \widetilde{\notin} s c l_{\widetilde{g}_{12}}\left(y_{\beta}, E\right)$. Therefore $x_{\alpha} \widetilde{\notin} s l_{\widetilde{g}_{12}}\left(y_{\beta}, E\right)$. So $x_{\alpha} \widetilde{\in}\left[\operatorname{scl}_{\widetilde{g}_{12}}\left(y_{\beta}, E\right)\right]^{c}$ is a soft open set. Thus $\left[\operatorname{scl}_{\widetilde{g}_{12}}\left(y_{\beta}, E\right)\right]^{c}$ is a soft open set containing $x_{\alpha}$ but not $y_{\beta}$. That is, $\left(X, \widetilde{g}_{1}, \widetilde{g}_{2}, E\right)$ is pairwise soft $T_{0}$-space.

Proposition 1. Let $\left(X, \widetilde{g}_{1}, \widetilde{g}_{2}, E\right)$ be a SBGT-space over $X$ and $\left(X, \widetilde{g}_{1}, E\right)$ and $\left(X, \widetilde{g}_{2}, E\right)$ be two soft generalized topological spaces. If $\left(X, \widetilde{g}_{1}, E\right)$ or $\left(X, \widetilde{g}_{2}, E\right)$ is soft $T_{0}-$ space, then $\left(X, \widetilde{g}_{1}, \widetilde{g}_{2}, E\right)$ is a pairwise soft bigeneralized $T_{0}$-space.

Proof. Suppose that each distinct soft points $x_{\alpha}, y_{\beta} \widetilde{\in} S S(X)_{E}$ and $\left(X, \widetilde{g}_{1}, E\right)$ is a soft $T_{0}$-space. Then there exist $(F, E) \in \widetilde{g}_{1}$ such that $x_{\alpha} \widetilde{\in}(F, E), y_{\beta} \widetilde{\notin}(F, E)$ or $x_{\alpha} \widetilde{\notin}(F, E), y_{\beta} \widetilde{\in}(F, E)$. Since $(F, E) \in \widetilde{g}_{1} \subseteq \widetilde{g}_{12}$, we obtain the requirement. Similarly it provide for $\left(X, \widetilde{g}_{2}, E\right)$ space. Thus, $\left(X, \widetilde{g}_{1}, \widetilde{g}_{2}, E\right)$ is pairwise soft bigeneralized $T_{0}-$ space.

Remark. The converse of Proposition-1 is not true in general. It is shown in following example.

Example 2. According to Example-1, $\left(X, \widetilde{g}_{1}, E\right)$ is not soft $T_{0}$-space. For $b_{e_{1}} \neq a_{e_{2}}$, there is no soft open set in $\widetilde{g}_{1}$ which contains one of points but not contains the other. Similarly, $\left(X, \widetilde{g}_{2}, E\right)$ is not soft $T_{0}-$ space. For $a_{e_{1}} \neq b_{e_{2}}$, there is no open soft set in $\widetilde{g}_{2}$ which contains one of points but not contains the other. Thus $\left(X, \widetilde{g}_{1}, \widetilde{g}_{2}, E\right)$ is a pairwise soft bigeneralized $T_{0}$-space but $\left(X, \widetilde{g}_{1}, E\right)$ or $\left(X, \widetilde{g}_{2}, E\right)$ is not soft $T_{0}$-space.

Definition 26. Let $\left(X, \widetilde{g}_{1}, \widetilde{g}_{2}, E\right)$ be a SBGT-space over $X$ and each distinct soft points $x_{\alpha}, y_{\beta} \widetilde{\in} S S(X)_{E}$. If there exist pairwise soft open sets $(F, E),(G, E) \in \widetilde{g}_{12}$ such that $x_{\alpha} \widetilde{\in}(F, E), y_{\beta} \widetilde{\notin}(F, E)$ and $y_{\beta} \widetilde{\in}(G, E), x_{\alpha} \widetilde{\notin}(G, E)$, then $\left(X, \widetilde{g}_{1}, \widetilde{g}_{2}, E\right)$ is called a pairwise soft bigeneralized $T_{1}-$ space.

Example 3. Let $X=\{a, b\}, E=\left\{e_{1}, e_{2}\right\}$ and let

$$
\begin{aligned}
& \widetilde{g}_{1}=\left\{(\widetilde{\phi}, E),\left(F_{1}, E\right),\left(F_{2}, E\right),\left(F_{3}, E\right)\right\} \\
& \widetilde{g}_{2}=\left\{(\widetilde{\phi}, E),\left(G_{1}, E\right),\left(G_{2}, E\right),\left(G_{3}, E\right)\right\}
\end{aligned}
$$


where

$$
\begin{aligned}
& \left(F_{1}, E\right)=\left\{\left(e_{1}, X\right),\left(e_{2}, \phi\right)\right\} \\
& \left(F_{2}, E\right)=\left\{\left(e_{1},\{a\}\right),\left(e_{2},\{b\}\right)\right\} \\
& \left(F_{3}, E\right)=\left\{\left(e_{1}, X\right),\left(e_{2},\{b\}\right\}\right. \\
& \left(G_{1}, E\right)=\left\{\left(e_{1}, \phi\right),\left(e_{2}, X\right)\right\} \\
& \left(G_{2}, E\right)=\left\{\left(e_{1},\{b\}\right),\left(e_{2},\{a\}\right)\right\} \\
& \left(G_{3}, E\right)=\left\{\left(e_{1},\{b\}\right),\left(e_{2}, X\right)\right\}
\end{aligned}
$$

Then, $\left(X, \widetilde{g}_{1}, \widetilde{g}_{2}, E\right)$ is a SBGT-space. Thus, the family of pairwise soft open sets over $\left(X, \widetilde{g}_{1}, \widetilde{g}_{2}, E\right)$ becomes

$$
\widetilde{g}_{12}=\left\{(\widetilde{\phi}, E),\left(F_{1}, E\right),\left(F_{2}, E\right),\left(F_{3}, E\right),\left(G_{1}, E\right),\left(G_{2}, E\right),\left(G_{3}, E\right),\left(H_{1}, E\right),\left(H_{2}, E\right),\left(H_{3}, E\right)\right\}
$$

where

$$
\begin{aligned}
& \left(H_{1}, E\right)=\left\{\left(e_{1}, X\right),\left(e_{2}, X\right)\right\} \\
& \left(H_{2}, E\right)=\left\{\left(e_{1}, X\right),\left(e_{2},\{a\}\right)\right\} \\
& \left(H_{1}, E\right)=\left\{\left(e_{1},\{a\}\right),\left(e_{2}, X\right)\right\}
\end{aligned}
$$

It is clear that $\left(X, \widetilde{g}_{1}, \widetilde{g}_{2}, E\right)$ is a pairwise soft bigeneralized $T_{1}$-space.

Theorem 4. Let $\left(X, \widetilde{g}_{1}, \widetilde{g}_{2}, E\right)$ be a SBGT-space over $X$. Then $\left(X, \widetilde{g}_{1}, \widetilde{g}_{2}, E\right)$ is a pairwise soft bigeneralized $T_{1}-$ space if and only if every single soft point set over $X$ is a pairwise soft closed set.

Proof. $(\Rightarrow)$ : Suppose that $\left(X, \widetilde{g}_{1}, \widetilde{g}_{2}, E\right)$ is pairwise soft bigeneralized $T_{0}$-space and each $x_{\alpha} \tilde{\in} S S(X)_{E}, y_{\beta} \widetilde{\in}\left(x_{\alpha}, E\right)^{c}$. Then there exist $(G, E) \in \widetilde{g}_{12}$ such that $y_{\beta} \widetilde{\in}(G, E)$ and $x_{\alpha} \widetilde{\notin}(G, E)$. Hence we obtain $y_{\beta} \widetilde{\in}(G, E) \subseteq\left(x_{\alpha}, E\right)^{c}$. Therefore $\left(x_{\alpha}, E\right)^{c}$ is a pairwise soft closed set. Thus $\left(x_{\alpha}, E\right)$ is a pairwise soft closed set.

$(\Leftarrow)$ : Suppose that for each distinct soft points $x_{\alpha}, y_{\beta} \widetilde{\in S S}(X)_{E}, \quad\left(x_{\alpha}, E\right)$ is a pairwise soft closed set then

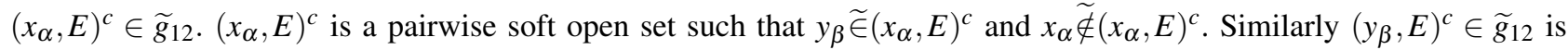
such that $x_{\alpha} \widetilde{\in}\left(y_{\beta}, E\right)^{c}$ and $y_{\beta} \widetilde{\notin}\left(y_{\beta}, E\right)^{c}$. Thus $\left(X, \widetilde{g}_{1}, \widetilde{g}_{2}, E\right)$ is a pairwise soft bigeneralized $T_{1}-$ space.

Proposition 2. Let $\left(X, \widetilde{g}_{1}, \widetilde{g}_{2}, E\right)$ be a SBGT-space over $X$ and $\left(X, \widetilde{g}_{1}, E\right)$ and $\left(X, \widetilde{g}_{2}, E\right)$ be two soft generalized topological spaces. If $\left(X, \widetilde{g}_{1}, E\right)$ or $\left(X, \widetilde{g}_{2}, E\right)$ is soft $T_{1}$-space, then $\left(X, \widetilde{g}_{1}, \widetilde{g}_{2}, E\right)$ is a pairwise soft bigeneralized $T_{1}-$ space.

Proof. Suppose that each distinct soft points $x_{\alpha}, y_{\beta} \widetilde{\in} S S(X)_{E}$ and $\left(X, \widetilde{g}_{1}, E\right)$ is a soft $T_{1}$-space. Then there exist $\left(F_{1}, E\right),\left(F_{2}, E\right) \in \widetilde{g}_{1}$ such that $x_{\alpha} \widetilde{\in}\left(F_{1}, E\right), y_{\beta} \widetilde{\notin}\left(F_{1}, E\right)$ and $x_{\alpha} \widetilde{\notin}\left(F_{2}, E\right), y_{\beta} \widetilde{\in}\left(F_{2}, E\right)$. Since $\left(F_{1}, E\right),\left(F_{2}, E\right) \in \widetilde{g}_{1} \subseteq \widetilde{g}_{12}$, we obtain the requirement. Similarly it is provided for $\left(X, \widetilde{g}_{2}, E\right)$ space. Thus, $\left(X, \widetilde{g}_{1}, \widetilde{g}_{2}, E\right)$ is pairwise soft bigeneralized $T_{1}$-space.

Remark. The converse of Proposition-2 is not true in general. It is shown in following example.

Example 4. According to Example-3, $\left(X, \widetilde{g}_{1}, \widetilde{g}_{2}, E\right)$ is a pairwise soft bigeneralized $T_{1}$-space. But $\left(X, \widetilde{g}_{1}, E\right)$ and $\left(X, \widetilde{g}_{2}, E\right)$ is not soft $T_{1}$-space. We consider $\left(X, \widetilde{g}_{1}, E\right)$ space. For $a_{e_{1}} \neq b_{e_{2}}$, there is no soft open set in $\widetilde{g}_{1}$ which contains $b_{e_{2}}$ but not contains $a_{e_{1}}$. Hence $\left(X, \widetilde{g}_{1}, E\right)$ is not soft $T_{1}-$ space. Similarly, we consider $\left(X, \widetilde{g}_{2}, E\right)$ space. For $b_{e_{1}} \neq a_{e_{2}}$, there is no soft open set in $\widetilde{g}_{2}$ which contains $b_{e_{1}}$ but not contains $a_{e_{2}}$. Hence $\left(X, \widetilde{g}_{2}, E\right)$ is not soft $T_{1}-$ space. Thus $\left(X, \widetilde{g}_{1}, \widetilde{g}_{2}, E\right)$ is a pairwise soft bigeneralized $T_{1}$-space but $\left(X, \widetilde{g}_{1}, E\right)$ or $\left(X, \widetilde{g}_{2}, E\right)$ is not soft $T_{0}$-space. 
Theorem 5. Every pairwise soft bigeneralized $T_{1}$-space is also a pairwise soft bigeneralized $T_{0}$-space.

Proof. Suppose that $\left(X, \widetilde{g}_{1}, \widetilde{g}_{2}, E\right)$ is pairwise soft bigeneralized $T_{1}$-space. Then for each distinct soft points $x_{\alpha}, y_{\beta} \widetilde{\in} S S(X)_{E}$, there exist $(F, E),(G, E) \in \widetilde{g}_{12}$ such that $x_{\alpha} \widetilde{\in}(F, E), y_{\beta} \widetilde{\notin}(F, E)$ and $y_{\beta} \widetilde{\in}(G, E), x_{\alpha} \widetilde{\notin}(G, E)$. Hence we obtain required conditions for pairwise soft bigeneralized $T_{0}$-space. Thus $\left(X, \widetilde{g}_{1}, \widetilde{g}_{2}, E\right)$ is a pairwise soft bigeneralized $T_{1}$-space.

Remark. The converse of Theorem-5 need not be true in general. It is shown in following example.

Example 5. According to Example-1, $\left(X, \widetilde{g}_{1}, \widetilde{g}_{2}, E\right)$ is a pairwise soft bigeneralized $T_{0}$-space. For $a_{e_{2}} \neq b_{e_{1}}$, there is no soft open set in $\widetilde{g}_{12}$ which contains $a_{e_{2}}$ but not contains $b_{e_{1}}$. Therefore $\left(X, \widetilde{g}_{1}, \widetilde{g}_{2}, E\right)$ is not pairwise soft bigeneralized $T_{1}$-space.

Definition 27. Let $\left(X, \widetilde{g}_{1}, \widetilde{g}_{2}, E\right)$ be a SBGT-space over $X$. If for each distinct soft points $x_{\alpha}, y_{\beta} \widetilde{\in} S S(X)_{E}$, there exist pairwise soft open sets $(U, E),(V, E) \in \widetilde{g}_{12}$ such that $(U, E) \widetilde{\cap}(V, E)=(\widetilde{\phi}, E)$, where $(U, E)$ and $(V, E)$ means that $x_{\alpha} \widetilde{\in}(U, E)$ and $y_{\beta} \widetilde{\in}(V, E)$, respectively, then $\left(X, \widetilde{g}_{1}, \widetilde{g}_{2}, E\right)$ is called a pairwise soft bigeneralized $T_{2}$-space.

Example 6. Consider a discrete SBGT-space $\left(X, \widetilde{g}_{1}, \widetilde{g}_{2}, E\right)$. Let $x_{\alpha}, y_{\beta} \widetilde{\in} S S(X)_{E}$ with $x_{\alpha} \neq y_{\beta} .\left\{x_{\alpha}\right\}$ and $\left\{y_{\beta}\right\}$ are soft open sets and $\left\{x_{\alpha}\right\} \cap\left\{y_{\beta}\right\}=(\widetilde{\phi} . E)$. Hence $\left(X, \widetilde{g}_{1}, \widetilde{g}_{2}, E\right)$ is a pairwise soft bigeneralized $T_{2}$-space.

Theorem 6. Let $\left(X, \widetilde{g}_{1}, \widetilde{g}_{2}, E\right)$ be a SBGT-space over $X$. Then $\left(X, \widetilde{g}_{1}, \widetilde{g}_{2}, E\right)$ is a pairwise soft bigeneralized $T_{2}-$ space if and only if there exist a soft open set $(U, E)$ containing $x_{\alpha}$ but not $y_{\beta}$ such that $y_{\beta} \widetilde{\notin} s c \widetilde{g}_{\tilde{g}_{12}}(V, E)$ for distinct point $x_{\alpha}, y_{\beta} \tilde{\in} S S(X)_{E}$.

Proof. $(\Rightarrow)$ : Let $\left(X, \widetilde{g}_{1}, \widetilde{g}_{2}, E\right)$ be a pairwise soft bigeneralized $T_{2}$-space and each distinct soft points $x_{\alpha}, y_{\beta} \widetilde{\in} S S(X)_{E}$. Then there exist disjoint pairwise soft open sets $(U, E)$ and $(V, E)$ such that $x_{\alpha} \widetilde{\in}(U, E)$ and $y_{\beta} \widetilde{\in}(V, E)$. This implies that $x_{\alpha} \tilde{\in}(U, E)^{c}$. So $(U, E)^{c}=(V, E)$ is a pairwise soft closed set containing $x_{\alpha}$ but not $y_{\beta}$ and $\operatorname{scl}_{\widetilde{g}_{12}}(U, E)=(V, E)$. Hence $y_{\beta} \widetilde{\notin} s l_{\tilde{g}_{12}}(U, E)$.

$(\Leftarrow)$ : Let $x_{\alpha}$ and $y_{\beta}$ be two distinct soft points in $\left(X, \widetilde{g}_{1}, \widetilde{g}_{2}, E\right)$. Then there exist a pairwise soft open set $(U, E)$ containing $x_{\alpha}$ but not $y_{\beta}$ such that $y_{\beta} \widetilde{\notin}_{s c \widetilde{g}_{12}}(V, E)$. This implies that $y_{\beta} \widetilde{\in}\left[\operatorname{scl}_{\widetilde{g}_{12}}(V, E)\right]^{c}$. Hence $(V, E)$ and $\left[\operatorname{scl}_{\widetilde{g}_{12}}(V, E)\right]^{c}$ are two disjoint pairwise soft open sets containing $x_{\alpha}$ and $y_{\beta}$ respectively and $(V, E) \widetilde{\cap}\left[s c l_{\widetilde{g}_{12}}(V, E)\right]^{c}=$ $(\widetilde{\phi}, E)$. Thus $\left(X, \widetilde{g}_{1}, \widetilde{g}_{2}, E\right)$ is pairwise soft bigeneralized $T_{2}$-space.

Proposition 3. Let $\left(X, \widetilde{g}_{1}, \widetilde{g}_{2}, E\right)$ be a SBGT-space over $X$ and $\left(X, \widetilde{g}_{1}, E\right)$ and $\left(X, \widetilde{g}_{2}, E\right)$ be two soft generalized topological spaces. If $\left(X, \widetilde{g}_{1}, E\right)$ or $\left(X, \widetilde{g}_{2}, E\right)$ is soft $T_{2}-$ space, then $\left(X, \widetilde{g}_{1}, \widetilde{g}_{2}, E\right)$ is a pairwise soft bigeneralized $T_{2}$-space.

Proof. Suppose that each distinct soft points $x_{\alpha}, y_{\beta} \widetilde{\in S S}(X)_{E}$ and $\left(X, \widetilde{g}_{1}, E\right)$ is a soft $T_{2}$-space. Then there exist $\left(F_{1}, E\right),\left(F_{2}, E\right) \in \widetilde{g}_{1}$ such that $\left(F_{1}, E\right) \widetilde{\cap}\left(F_{2}, E\right)=(\widetilde{\phi}, E)$, where $\left(F_{1}, E\right)$ and $\left(F_{2}, E\right)$ means that $x_{\alpha} \widetilde{\in}\left(F_{1}, E\right)$ and $y_{\beta} \widetilde{\in}\left(F_{2}, E\right)$, respectively. Since $\left(F_{1}, E\right),\left(F_{2}, E\right) \in \widetilde{g}_{1} \subseteq \widetilde{g}_{12}$, we obtain the requirement. Similarly, it is provided for $\left(X, \widetilde{g}_{2}, E\right)$ space. Thus $\left(X, \widetilde{g}_{1}, \widetilde{g}_{2}, E\right)$ is a pairwise soft bigeneralized $T_{2}-$ space.

Theorem 7. Every pairwise soft $T_{2}-$ space is pairwise soft $T_{1}-$ space.

Proof. Suppose that $\left(X, \widetilde{g}_{1}, \widetilde{g}_{2}, E\right)$ be a SBGT-space over $X$ and each distinct soft points $x_{\alpha}, y_{\beta} \widetilde{\in} S S(X)_{E}$. If $\left(X, \widetilde{g}_{1}, \widetilde{g}_{2}, E\right)$ is pairwise soft $T_{2}$-space, then $\left(X, \widetilde{g}_{1}, E\right)$ or $\left(X, \widetilde{g}_{2}, E\right)$ is a soft $T_{2}$-space. If $\left(X, \widetilde{g}_{1}, E\right)$ is soft $T_{2}$-space, then there exist a

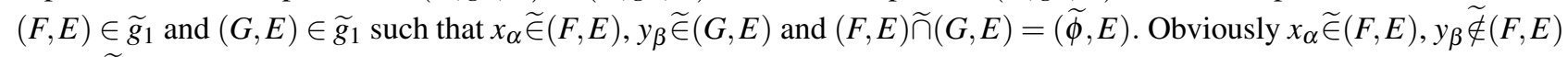
and $x_{\alpha} \tilde{\notin}(G, E), y_{\beta} \widetilde{\in}(G, E)$. Therefore $\left(X, \widetilde{g}_{1}, E\right)$ is a soft $T_{1}$-space. According to Proposition-2, $\left(X, \widetilde{g}_{1}, \widetilde{g}_{2}, E\right)$ is pairwise soft bigeneralized $T_{1}$-space.

Remark. The converse of Theorem-7 is not true in general. It is shown in following example. 
Example 7. According to Example-3, $\left(X, \widetilde{g}_{1}, \widetilde{g}_{2}, E\right)$ pairwise soft bigeneralized $T_{1}$-space but not pairwise soft bigeneralized $T_{2}$-space. For $a_{e_{2}} \neq b_{e_{1}}$, there is no soft open sets in $\widetilde{g}_{12}$ such that $a_{e_{2}} \widetilde{\in}(U, E), b_{e_{1}} \widetilde{\epsilon}(V, E)$ and $(U, E) \widetilde{\cap}(V, E)=(\widetilde{\phi}, E)$. Thus $\left(X, \widetilde{g}_{1}, \widetilde{g}_{2}, E\right)$ is not pairwise soft bigeneralized $T_{2}$-space.

Theorem 8. The property of being pairwise soft bigeneralized $T_{i}-$ space $(i=0,1,2)$ is preserved under a soft $\widetilde{g}_{12}$-homeomorphism mapping.

Proof. We prove the theorem for $i=2$, the other cases is similar. Let $f:\left(X, \widetilde{g}_{1}, \widetilde{g}_{2}, E\right) \rightarrow\left(Y, \widetilde{k}_{1}, \widetilde{k}_{2}, E\right)$ be a soft mapping such that:

(1) $\mathrm{f}$ is $1-1$, onto and soft open mapping.

(2) $\left(X, \widetilde{g}_{1}, \widetilde{g}_{2}, E\right)$ is a pairwise soft bigeneralized $T_{2}$-space.

We want to show that $\left(Y, \widetilde{k}_{1}, \widetilde{k}_{2}, E\right)$ is a pairwise soft bigeneralized $T_{2}-$ space. So, let $x_{\alpha}, y_{\beta} \widetilde{\in S S}(Y)_{E}$ such that $x_{\alpha} \neq y_{\beta}$. Since $\mathrm{f}$ is $1-1$ and onto mapping, then there exist two soft points $x_{\alpha}^{\prime}, y_{\beta}^{\prime}$ in $\widetilde{X}$ such that $f\left(x_{\alpha}^{\prime}\right)=x_{\alpha}, f\left(y_{\beta}^{\prime}\right)=y_{\beta}$, and $x_{\alpha}^{\prime} \neq y_{\beta}^{\prime}$. But $\left(X, \widetilde{g}_{1}, \widetilde{g}_{2}, E\right)$ is a pairwise soft $T_{2}$-space, so, there exist $(G, E),(H, E) \in \widetilde{g}_{12}$ such that $x_{\alpha}^{\prime} \widetilde{\in}(G, E)$, $y_{\beta}^{\prime} \tilde{\in}(H, E)$ and $(G, E) \widetilde{\cap}(H, E)=(\widetilde{\phi}, E)$. It follows that, $f\left(x_{\alpha}^{\prime}\right)=x_{\alpha} \widetilde{\in} f((G, E)), f\left(y_{\beta}^{\prime}\right)=y_{\beta} \widetilde{\in} f((H, E))$ and $f((G, E) \widetilde{\cap}(H, E))=f((G, E)) \widetilde{\cap} f((H, E))=f((\widetilde{\phi}, E))=(\widetilde{\phi}, E)$. Since $(G, E),(H, E) \in \widetilde{g}_{12}$ and $\mathrm{f}$ is an soft open mapping, $f((G, E)), f((H, E)) \in \widetilde{k}_{12}$. Then, there exist $f((G, E)), f((H, E)) \in \widetilde{k}_{12}$ such that $x_{\alpha} \widetilde{\in} f((G, E))$, $y_{\beta} \widetilde{\in} f((H, E))$ and $f((G, E)) \widetilde{\cap} f((H, E))=(\widetilde{\phi}, E)$. Hence $\left(Y, \widetilde{k}_{1}, \widetilde{k}_{2}, E\right)$ is a pairwise soft bigeneralized $T_{2}$-space

Definition 28. Let $\left(X, \widetilde{g}_{1}, \widetilde{g}_{2}, E\right)$ be a SBGT-space and pairwise soft bigeneralized $T_{1}-$ space over $X,(F, E)$ be a soft closed set in $X$ and $x_{\alpha} \widetilde{\in} S S(X)_{E}$ such that $x_{\alpha} \tilde{\notin}(F, E)$. If there exist $\left(G_{1}, E\right),\left(G_{2}, E\right) \in \widetilde{g}_{12}$ such that $x_{\alpha} \widetilde{\in}\left(G_{1}, E\right),(F, E) \widetilde{C}\left(G_{2}, E\right)$ and $\left(G_{1}, E\right) \widetilde{\cap}\left(G_{2}, E\right)=(\widetilde{\phi}, E)$, then $\left(X, \widetilde{g}_{1}, \widetilde{g}_{2}, E\right)$ is said to be a pairwise soft bigeneralized $T_{3}$-space.

Definition 29. Let $\left(X, \widetilde{g}_{1}, \widetilde{g}_{2}, E\right)$ be a SBGT-space and pairwise soft bigeneralized $T_{1}$-space over $X,(F, E)$ and $(G, E)$ soft closed sets over $X$ such that $(F, E) \widetilde{\cap}(G, E)=(\widetilde{\phi}, E)$. If there exist $\left(F_{1}, E\right),\left(F_{2}, E\right) \in \widetilde{g}_{12}$ such that $(F, E) \widetilde{C}\left(F_{1}, E\right)$, $(G, E) \widetilde{C}\left(F_{2}, E\right)$ and $\left(F_{1}, E\right) \widetilde{\cap}\left(F_{2}, E\right)=\widetilde{\phi}$, then $\left(X, \widetilde{g}_{1}, \widetilde{g}_{2}, E\right)$ is called a pairwise soft bigeneralized $T_{4}-$ space.

Theorem 9. The property of being pairwise soft bigeneralized $T_{i}$-space $(i=3,4)$ is preserved under a soft $\widetilde{g}_{12}$-homeomorphism mapping.

Proof. We prove the theorem for $i=3$, the other cases are similar. So, let $f:\left(X, \widetilde{g}_{1}, \widetilde{g}_{2}, E\right) \rightarrow\left(Y, \widetilde{k}_{1}, \widetilde{k}_{2}, E\right)$ be a soft mapping such that:

(1) $f:\left(X, \widetilde{g}_{1}, \widetilde{g}_{2}, E\right) \rightarrow\left(Y, \widetilde{k}_{1}, \widetilde{k}_{2}, E\right)$ is soft $\widetilde{g}_{12}$-homeomorphism (i.e $f$ is a soft bijection and $f, f^{-1}$ are soft $\widetilde{g}_{12}$-continuous)

(2) $\left(X, \widetilde{g}_{1}, \widetilde{g}_{2}, E\right)$ is a pairwise soft bigeneralized $T_{3}$-space.

Let $(F, E)$ be a $\widetilde{k}_{12}$-closed soft subset of $Y$ and let $y_{\beta} \widetilde{\in} S S(Y)_{E}$ such that $y_{\beta} \widetilde{\notin}(F, E)$. Since, $f$ is an onto mapping, there exist $x_{\alpha} \tilde{\in} S S(X)_{E}$ such that $f\left(x_{\alpha}\right)=y_{\beta}$.

Since $f$ is soft continuous mapping and $(F, E)$ is a $\widetilde{k}_{12}$-closed soft subset of $Y$, we have $f^{-1}((F, E))$ is a $\widetilde{g}_{12}-$ closed soft subset of $X$. Since $y_{\beta}=f\left(x_{\alpha}\right) \widetilde{\notin}(F, E)$, we have $f^{-1}\left(f\left(x_{\alpha}\right)\right)=x_{\alpha} \widetilde{\notin} f^{-1}((F, E))$ (as, $f$ is soft injective). Now, $f^{-1}((F, E))$ is a $\widetilde{g}_{12}$-soft closed subset of $X, x_{\alpha} \widetilde{\in} S S(X)_{E}$ such that $x_{\alpha} \notin f^{-1}((F, E))$. Moreover $\left(X, \widetilde{g}_{1}, \widetilde{g}_{2}, E\right)$ is a pairwise soft $T_{3}$-space, so, there exist $(G, E),(H, E) \in \widetilde{g}_{12}$ such that $x_{\alpha} \widetilde{\in}(G . E), \quad f^{-1}((F, E)) \widetilde{\subseteq}(H, E)$ and $(G, E) \widetilde{\cap}(H, E)=(\widetilde{\phi}, E)$ and therefore $f\left(x_{\alpha}\right)=y_{\beta} \widetilde{\in} f((G, E)), f\left(f^{-1}((F, E))\right)=(F, E) \widetilde{\subseteq} f((H ; E))$ (as, $f$ is soft surjective) and $f((G, E) \widetilde{\cap}(H, E))=f((G, E)) \widetilde{\cap} f((H, E))=f((\widetilde{\phi}, E))=(\widetilde{\phi}, E)$.

Since $f^{-1}$ is soft $\widetilde{g}_{12}$-continuous mapping, i.e. $f$ is an soft open mapping (from Theorem-2). If $(G, E),(H, E) \in \widetilde{g}_{12}$ and $f$ is a soft open mapping, then $f((G, E)), f((H, E)) \in \widetilde{k}_{12}$ (from Definition-23). Therefore, there exist $f((\underset{\widetilde{k}}{\tilde{\sim}}, E)), f((H, E)) \in \widetilde{k}_{12}$ such that $y_{\beta} \widetilde{\in} f((G, E)),(F, E) \widetilde{\subseteq} f((H, E))$ and $f((G, E)) \widetilde{\cap} f((H, E))=(\widetilde{\phi}, E)$. Thus, $\left(Y, \widetilde{k}_{1}, \widetilde{k}_{2}, E\right)$ is a pairwise soft bigeneralized $T_{3}-$ space. 


\section{Soft hereditary property}

Definition 30. Let $\left(X, \widetilde{g}_{1}, \widetilde{g}_{2}, E\right)$ be a SBGT-space over $X$ and $Y$ be a non-empty subset of $X$. Then $\widetilde{g}_{1 Y}=\left\{\left({ }^{Y} F, E\right):(F, E) \in\right.$ $\left.\widetilde{g}_{1}\right\}$ and $\widetilde{g}_{2 Y}=\left\{\left({ }^{Y} G, E\right):(G, E) \in \widetilde{g}_{2}\right\}$ are said to be the relative soft topologies on $\widetilde{Y}$. Moreover $\left(Y, \widetilde{g}_{1 Y}, \widetilde{g}_{2 Y}, E\right)$ is called a soft bigeneralized subspace of $\left(X, \widetilde{g}_{1}, \widetilde{g}_{2}, E\right)$.

Theorem 10. Let $\left(X, \widetilde{g}_{1}, \widetilde{g}_{2}, E\right)$ be a SBGT-space over $X$ and $Y$ be a non-empty subset of $X$. Then $\left(Y, \widetilde{g}_{1 Y}, \widetilde{g}_{2 Y}, E\right)$ is a SBGT-space on Y. Moreover $\widetilde{g}_{1 Y 2 Y}=\widetilde{g}_{12 Y}$, where $\widetilde{g}_{12 Y}=\left\{(Y, E) \widetilde{\cap}(G, E):(G, E) \in \widetilde{g}_{12}\right\}$ and $\widetilde{g}_{1 Y 2 Y}=\left\{(H, E) \in S S(Y)_{E}\right.$ : $\left.(H, E)=\left(H_{1}, E\right) \widetilde{\cup}\left(H_{2}, E\right),\left(H_{i}, E\right) \in \widetilde{g}_{i Y}, i=1,2\right\}$.

Proof. Since $\left(X, \widetilde{g}_{1}, \widetilde{g}_{2}, E\right)$ is a SBGT-space, $\left(X, \widetilde{g}_{1}, E\right)$ and $\left(X, \widetilde{g}_{2}, E\right)$ are soft generalized topologies on $X$. Since $Y \subseteq X$, $\left(X, \widetilde{g}_{1 Y}, E\right)$ and $\left(X, \widetilde{g}_{2 Y}, E\right)$ are soft generalized topologies on Y. Consequently, $\left(Y, \widetilde{g}_{1 Y}, \widetilde{g}_{2 Y}, E\right)$ is a SBGT-space on $Y$.

Let $(G, E) \in \widetilde{g}_{12 Y}$. Then, there exist $(H, E) \in \widetilde{g}_{12}$ such that

$$
\begin{aligned}
(G, E) & =(Y, E) \widetilde{\cap}(H, E) \\
& =(Y, E) \widetilde{\cap}\left[\left(H_{1}, E\right) \widetilde{\cup}\left(H_{2}, E\right)\right],\left(H_{1}, E\right) \in \widetilde{g}_{1} \quad \text { and } \quad\left(H_{2}, E\right) \in \widetilde{g}_{2} \\
& =\left[(Y, E) \widetilde{\cap}\left(H_{1}, E\right)\right] \widetilde{\cup}\left[(Y, E) \widetilde{\cap}\left(H_{2}, E\right] .\right.
\end{aligned}
$$

Since $(Y, E) \widetilde{\cap}\left(H_{1}, E\right) \in \widetilde{g}_{1 Y}$ and $(Y, E) \widetilde{\cap}\left(H_{2}, E\right) \in \widetilde{g}_{2 Y},\left[(Y, E) \widetilde{\cap}\left(H_{1}, E\right)\right] \widetilde{\cup}\left[(Y, E) \widetilde{\cap}\left(H_{2}, E\right] \in \widetilde{g}_{1 Y 2 Y}\right.$. Thus, $(G, E) \in \widetilde{g}_{1 Y 2 Y}$. So, $\widetilde{g}_{12 Y} \subseteq \widetilde{g}_{1 Y 2 Y}$.

By similar way, we can prove that $\widetilde{g}_{1 Y 2 Y} \subseteq \widetilde{g}_{12 Y}$.

Proposition 4. Let $\left(X, \widetilde{g}_{1}, \widetilde{g}_{2}, E\right)$ be a SBGT-space over $X$ and $Y$ be a non-empty subset of $X$. If $\left(X, \widetilde{g}_{1}, \widetilde{g}_{2}, E\right)$ is pairwise soft bigeneralized $T_{0}-$ space then $\left(Y, \widetilde{g}_{1 Y}, \widetilde{g}_{2 Y}, E\right)$ is pairwise soft bigeneralized $T_{0}-$ space.

Proof. Suppose that $\left(X, \widetilde{g}_{1}, \widetilde{g}_{2}, E\right)$ be a pairwise soft bigeneralized $T_{0}$-space over $X$ and $\left(Y, \widetilde{g}_{1 Y}, \widetilde{g}_{2 Y}, E\right)$ be a soft bigeneralized subspace of $\left(X, \widetilde{g}_{1}, \widetilde{g}_{2}, E\right)$. Assume that each distinct soft points $x_{\alpha}, y_{\beta} \widetilde{\in} S S(Y)_{E}$. Since $Y \subseteq X$, $x_{\alpha}, y_{\beta} \widetilde{\in} S S(X)_{E}$. So, there exist some pairwise soft set $(F, E) \in \widetilde{g}_{12}$ such that $x_{\alpha} \widetilde{\in}(F, E)$ and $y_{\beta} \widetilde{\notin}(F, E)$ or $x_{\alpha} \widetilde{\notin}(F, E)$ and $y_{\beta} \widetilde{\in}(F, E)$. Then $x_{\alpha} \widetilde{\epsilon}(Y, E) \cap(F, E)=\left({ }^{Y} F, E\right)$ and $y_{\beta} \widetilde{\notin}(Y, E) \cap(F, E)=\left({ }^{Y} F, E\right)$ or $x_{\alpha} \widetilde{\notin}(Y, E) \cap(F, E)=\left({ }^{Y} F, E\right)$ and $y_{\beta} \widetilde{\in}(Y, E) \cap(F, E)=\left({ }^{Y} F, E\right)$. Since $\left({ }^{Y} F, E\right) \in \widetilde{g}_{12 Y},\left(Y, \widetilde{g}_{1 Y}, \widetilde{g}_{2 Y}, E\right)$ is a pairwise soft bigeneralized $T_{0}$-space.

Proposition 5. Let $\left(X, \widetilde{g}_{1}, \widetilde{g}_{2}, E\right)$ be a SBGT-space over $X$ and $Y$ be a non-empty subset of $X$. If $\left(X, \widetilde{g}_{1}, \widetilde{g}_{2}, E\right)$ is pairwise soft bigeneralized $T_{1}-$ space then $\left(Y, \widetilde{g}_{1 Y}, \widetilde{g}_{2 Y}, E\right)$ is pairwise soft bigeneralized $T_{1}-$ space.

Proof. Suppose that $\left(X, \widetilde{g}_{1}, \widetilde{g}_{2}, E\right)$ be a pairwise soft bigeneralized $T_{0}$-space over $X$ and $\left(Y, \widetilde{g}_{1 Y}, \widetilde{g}_{2 Y}, E\right)$ be a soft bigeneralized subspace of $\left(X, \widetilde{g}_{1}, \widetilde{g}_{2}, E\right)$. Assume that each distinct soft points $x_{\alpha}, y_{\beta} \widetilde{\in} S S(Y)_{E}$. Since $Y \subseteq X$, $x_{\alpha}, y_{\beta} \widetilde{\in} S S(X)_{E}$. So, there exist some pairwise soft sets $(F, E),(G, E) \in \widetilde{g}_{12}$ such that $x_{\alpha} \widetilde{\in}(F, E), y_{\beta} \widetilde{\notin}(F, E)$ and $x_{\alpha} \widetilde{\notin}(G, E), \quad y_{\beta} \widetilde{\in}(G, E) . \quad$ Then $\quad x_{\alpha} \widetilde{\in}(Y, E) \cap(F, E)=\left({ }^{Y} F, E\right), \quad y_{\beta} \widetilde{\notin}(Y, E) \cap(F, E)=\left({ }^{Y} F, E\right) \quad$ and $x_{\alpha} \widetilde{\notin}(Y, E) \cap(G, E)=\left({ }^{Y} G, E\right), y_{\beta} \widetilde{\in}(Y, E) \cap(G, E)=\left({ }^{Y} G, E\right)$. Since $\left({ }^{Y} F, E\right),\left({ }^{Y} G, E\right) \in \widetilde{g}_{12 Y},\left(Y, \widetilde{g}_{1 Y}, \widetilde{g}_{2 Y}, E\right)$ is a pairwise soft bigeneralized $T_{1}$-space.

Proposition 6. Let $\left(X, \widetilde{g}_{1}, \widetilde{g}_{2}, E\right)$ be a SBGT-space over $X$ and $Y$ be a non-empty subset of $X$. If $\left(X, \widetilde{g}_{1}, \widetilde{g}_{2}, E\right)$ is pairwise soft bigeneralized $T_{2}$-space then $\left(Y, \widetilde{g}_{1 Y}, \widetilde{g}_{2 Y}, E\right)$ is pairwise soft bigeneralized $T_{2}-$ space.

Proof. Suppose that $\left(X, \widetilde{g}_{1}, \widetilde{g}_{2}, E\right)$ be a pairwise soft bigeneralized $T_{0}$-space over $X$ and $\left(Y, \widetilde{g}_{1 Y}, \widetilde{g}_{2 Y}, E\right)$ be a soft bigeneralized subspace of $\left(X, \widetilde{g}_{1}, \widetilde{g}_{2}, E\right)$. Assume that each distinct soft points $x_{\alpha}, y_{\beta} \widetilde{\in} S S(Y)_{E}$. Since $Y \subseteq X$, 
$x_{\alpha}, y_{\beta} \widetilde{\in} S S(X)_{E}$. So, there exist $(F, E),(G, E) \in \widetilde{g}_{12}$ such that $x_{\alpha} \widetilde{\in}(F, E), y_{\beta} \widetilde{\in}(G, E)$ and $(F, E) \cap(G, E)=(\widetilde{\phi}, E)$. Moreover there exists $(Y, E) \cap(G, E),(Y, E) \cap(F, E) \in \widetilde{g}_{12 Y}$ such that $x_{\alpha} \widetilde{\in}(Y, E) \cap(F, E), y_{\beta} \widetilde{\in}(Y, E) \cap(G, E)$ and $[(Y, E) \cap(F, E)] \cap[(Y, E) \cap(G, E)]=(Y, E) \cap[(F, E) \cap(G, E)]=(Y, E) \cap(\widetilde{\phi}, E)=(\widetilde{\phi}, E)$. Hence $\left(Y, \widetilde{g}_{1 Y}, \widetilde{g}_{2 Y}, E\right)$ is a pairwise soft bigeneralized $T_{2}$-space.

Proposition 7. Let $\left(X, \widetilde{g}_{1}, \widetilde{g}_{2}, E\right)$ be a SBGT-space over $X$ and $Y$ be a non-empty subset of $X$. If $\left(X, \widetilde{g}_{1}, \widetilde{g}_{2}, E\right)$ is pairwise soft bigeneralized $T_{3}-$ space then $\left(Y, \widetilde{g}_{1 Y}, \widetilde{g}_{2 Y}, E\right)$ is pairwise soft bigeneralized $T_{3}-$ space.

Proof. Let $y_{\beta} \widetilde{\in} S S(Y)_{E}$ and $(F, E)$ be a soft closed set in $\widetilde{Y}$ such that $y_{\beta} \widetilde{\notin}(F, E)$. Then $y_{\beta} \widetilde{\notin}((Y, E) \widetilde{\cap}(G, E))$ where $(F, E)=((Y, E) \widetilde{\cap}(G, E))$ for some soft closed set in $\widetilde{X}$. But $y_{\beta} \widetilde{\in}(Y, E)$, so $y_{\beta} \widetilde{\notin}(G, E)$. As $\left(X, \widetilde{g}_{1}, \widetilde{g}_{2}, E\right)$ is a pairwise soft $T_{3}$-space, so there exist $\left(G_{1}, E\right),\left(G_{2}, E\right) \widetilde{\in} \widetilde{g}_{12}$ such that $y_{\beta} \widetilde{\in}\left(G_{1}, E\right),(G, E) \widetilde{C}\left(G_{2}, E\right)$ and $\left(G_{1}, E\right) \widetilde{\cap}\left(G_{2}, E\right)=(\widetilde{\phi}, E)$. Now if we take $\left(F_{1}, E\right)=(Y, E) \widetilde{\cap}\left(G_{1}, E\right)$ and $\left(F_{2}, E\right)=(Y, E) \widetilde{\cap}\left(G_{2}, E\right)$, then $\left(F_{1}, E\right),\left(F_{2}, E\right) \in \widetilde{g}_{12 Y}$ such that $y_{\beta} \widetilde{\in}\left(F_{1}, E\right)$ and $(F, E) \widetilde{\subset}(Y, E) \widetilde{\cap}$

$\left(G_{2}, E\right)=\left(F_{2}, E\right)$ and $\left(F_{1}, E\right) \widetilde{\cap}\left(F_{2}, E\right) \widetilde{C}\left(G_{1}, E\right) \widetilde{\cap}\left(G_{2}, E\right)=(\widetilde{\phi}, E)$. Thus $\left(Y, \widetilde{g}_{1 Y}, \widetilde{g}_{2 Y}, E\right)$ is a soft bigeneralized $T_{3}-$ space.

Proposition 8. Every closed soft subspace of a pairwise soft $T_{4}-$ space is a pairwise soft bigeneralized $T_{4}-$ space.

Proof. Let $\left(X, \widetilde{g}_{1}, \widetilde{g}_{2}, E\right)$ be a pairwise soft bigeneralized $T_{4}$-space and $\left(Y, \widetilde{g}_{1 Y}, \widetilde{g}_{2 Y}, E\right)$ be a soft closed subspace of $\left(X, \widetilde{g}_{1}, \widetilde{g}_{2}, E\right)$. We want to show that $\left(Y, \widetilde{g}_{1 Y}, \widetilde{g}_{2 Y}, E\right)$ is a pairwise soft bigeneralized $T_{4}-$ space. So, let $\left(K_{1}, E\right),\left(K_{2}, E\right)$ be a non-empty disjoint closed soft subsets of $\widetilde{Y}$. Since $\left(K_{1}, E\right),\left(K_{2}, E\right) \in \widetilde{g}_{12 Y}^{c}$, there exists $\left(F_{1}, E\right),\left(F_{2}, E\right) \in \widetilde{g}_{12}^{c}$ such that $\left(K_{1}, E\right)=(Y, E) \widetilde{\cap}\left(F_{1}, E\right)$ and $\left(K_{2}, E\right)=(Y, E) \widetilde{\cap}\left(F_{2}, E\right)$. Since $(Y, E) \in \widetilde{g}_{12}^{c}$ and $\left(F_{1}, E\right),\left(F_{2}, E\right) \in \widetilde{g}_{12}^{c}$, we have $(Y, E) \widetilde{\cap}\left(F_{1}, E\right),(Y, E) \widetilde{\cap}\left(F_{2}, E\right) \in \widetilde{g}_{12}^{c}$.

Now $\left(K_{1}, E\right),\left(K_{2}, E\right)$ are two non-empty disjoint soft closed subset of $\widetilde{X}$, but $\left(X, \widetilde{g}_{1}, \widetilde{g}_{2}, E\right)$ is a pairwise soft $T_{4}-$ space, so, $(G, E),(H, E) \in \widetilde{g}_{12}$ such that $\left(K_{1}, E\right) \widetilde{\subseteq}(G, E),\left(K_{2}, E\right) \widetilde{\subseteq}(H, E)$ and $(G, E) \widetilde{\cap}(H, E)=(\widetilde{\phi}, E)$. It follows that, $\exists(Y, E) \widetilde{\cap}(G, E),(Y, E) \widetilde{\cap}(H, E) \in \widetilde{g}_{12 Y}$ such that $\left(K_{1}, E\right) \widetilde{\subseteq}(Y, E) \widetilde{\cap}(G, E),\left(K_{2}, E\right) \widetilde{\subseteq}(Y, E) \widetilde{\cap}(H, E)$ and

$((Y, E) \widetilde{\cap}(G, E)) \widetilde{\cap}((Y, E) \widetilde{\cap}(H, E))=(Y, E) \widetilde{\cap}((G, E) \widetilde{\cap}(H, E))=(Y, E) \widetilde{\cap}(\phi, E)=(\widetilde{\phi}, E)$. Therefore $\left(Y, \widetilde{g}_{1 Y}, \widetilde{g}_{2 Y}, E\right)$ is a pairwise soft bigeneralized $T_{4}$-space.

\section{Conclusion}

Soft bigeneralized topological spaces was introduced by T.Y. Ozturk et. al. [19] in 2018. A soft bigeneralized topological spaces is an important generalization of the bigeneralized topological spaces. Hence in this paper we introduced pairwise soft bigeneralized $T_{i}$-spaces $(i=0,1,2,3,4)$. Characterizations of these spaces are obtained. Finally, we showed that soft hereditary properties of these pairwise soft separation axioms. We hope that the findings in this paper will help researcher enhance and promote the further study on SBGT-spaces.

\section{Competing interests}

The authors declare that they have no competing interests.

\section{Authors' contributions}

All authors have contributed to all parts of the article. All authors read and approved the final manuscript. 


\section{References}

[1] S. Bayramov and C. Gunduz, Soft locally compact spaces and soft paracompact spaces, Journal of Math. and Sys. Sci. 3, 122-130 (2013).

[2] Á. Császár, Generalized topology, generalized continuity, Acta. Math. Hungar. 96(4), 351-357 (2002).

[3] Á. Császár, Generalized open sets, Acta. Math. Hungar. 75, (1-2), 65-87 (1997).

[4] N. Cağman N, S. Karataş and S. Enginoğlu, Soft topology, Comput. Math. Appl. 351-358 (2011).

[5] C. Gunduz Aras, A. Sonmez and H. Cakall1, An approach to soft functions. Journal of Mathematical Analysis, 8(2), (2017)

[6] C. Gunduz (Aras), A. Yolcu, T. Y. Ozturk, A study on soft generalized continuity in soft bigeneralized topological spaces, Journal of Mathematical Analysis, 9(5), 106-118 (2018).

[7] S. Hussain and B. Ahmad, Some properties of soft topological spaces, Comput. Math. Appl. 62, 4058-4067 (2011).

[8] B.M. Ittanagi, Soft bitopological spaces, Int. Journal of Computer Applications 107(7), 1-4 (2014).

[9] A. Kandil, O.A.E. Tantawy, S.A. El-Sheikh and S.A. Hazza, Pairwise open (closed) soft sets in soft bitopological spaces, Annals of Fuzzy Math. and Infor. 11(4), 571-588 (2016).

[10] J.C. Kelly, Bitopological spaces, Proc. London Math. Soc., (3)13, 71-89 (1963).

[11] A. Kharal and B. Ahmad, Mapping on soft classes, New Math. \& Natural Comput. 7(3), 471-481 (2011).

[12] N. Levine, Semi-open sets and semi-continuity in topological spaces, Amer. Math. Monthly, 70, 36-41 (1963).

[13] P.K. Maji and A.R. Roy, An application of soft sets in a decision making problem, Comput. Math. Appl. 44, 1077-1083 (2002).

[14] P.K. Maji, R. Biswas and A.R. Roy, Soft set theory, Comput. Math. Appl. 45, 555-562 (2003).

[15] W.K. Min, A note on soft topological spaces, Comput. Math. Appl. 62, 3524-3528 (2011).

[16] W.K. Min and Y. K. Kim, Quasi generalized open sets and quasi generalized continuity on bigeneralized topological spaces, Honam Mathematical J. 32(4), 619-624 (2010).

[17] D. Molodtsov, Soft set theory-first results, Comput. Math. Appl. 37, 19-31 (1999).

[18] T.Y Ozturk and S. Bayramov, Soft mapping spaces, The Scientific World Journal, Article ID 307292, (2014) 8p.

[19] T.Y Ozturk, C. Gunduz(Aras), A. Yolcu, Soft Bigeneralized Topological Spaces, Filomat, 32(16), 5679-5690 (2018).

[20] M. Shabir M and M. Naz, On soft topological spaces, Comput. Math. Appl. 61, 1786-1799 (2011).

[21] M. Shabir and A. Bashir, Some properties of soft topological spaces, Comput. Math. Appl. 62, 4058-4067 (2011).

[22] J. Thomas and J. S. John, On soft generalized topological spaces, Journ. of New Results in Sci. 4, 01-15 (2014).

[23] P.Torton, C. Viriyapong and C. Boonpok, Some separation axioms in bigeneralized topological spaces, Int. Journal of Math. Analysis, 6(56), 2789-2796 (2012).

[24] I Zorlutuna, M. Akdağ, W.K. Min and S. Atmaca, Remarks on soft topological spaces, Annals of Fuzzy Math. and Infor. 3(2), 171-185 (2012). 